\title{
Diagnostic accuracy of magnetic resonance imaging in the evaluation of pulmonary infections in immunocompromised patients
}

\author{
Rashmi Singh ${ }^{A, B, C, D, E, F}$, Mandeep Garg ${ }^{A, B, C, D, E, F}$, Kushaljit S. Sodhi ${ }^{A, B, D, E, F}$, Nidhi Prabhakar ${ }^{A, C, D, E, F}$, \\ Paramjeet Singh ${ }^{A, B, D, E}$, Ritesh Agarwal ${ }^{A, B, D, E}$, Pankaj Malhotra ${ }^{A, B, D, E}$ \\ Post Graduate Institute of Medical Education and Research, Chandigarh, India
}

\section{Abstract}

Purpose: To evaluate the accuracy of magnetic resonance imaging (MRI) for diagnosing pulmonary infections in immunocompromised adults.

\begin{abstract}
Material and methods: Computed tomography (CT) and MRI chest were performed in 35 immuno-compromised patients suspected of pulmonary infection. The MRI sequences that were performed included axial and coronal T2 half-Fourier acquisition single-shot turbo spin-echo (HASTE), spectrally attenuated inversion recovery (SPAIR), true fast imaging with steady-state free precession (TRUFI), and three-dimensional fast low angle shot (3D FLASH) using breath-hold and respiratory triggered BLADE (proprietary name for periodically rotated overlapping parallel lines with enhanced reconstruction). The presence of nodules, consolidations, and ground-glass opacities was evaluated. Sensitivity, specificity, positive predictive value (PPV), and negative predictive value (NPV) were calculated for MRI using CT scan as a reference standard.
\end{abstract}

Results: The sensitivity of MRI in nodule detection was $50 \%$ overall and $75 \%$ for nodules measuring more than $5 \mathrm{~mm}$. Consolidation was detected with $100 \%$ sensitivity. Sensitivity and PPV for the detection of ground-glass opacities (GGOs) were $77.7 \%$ and $53.8 \%$, respectively. T2 HASTE axial had the fewest image artefacts. Respiratory triggered MR pulse sequence did not add any significant diagnostic information as compared to the non-respiratory triggered MR pulse sequences.

Conclusions: Sensitivity for detecting small nodules and GGOs on MR is poor; CT scan remains the imaging modality of choice for the evaluation of pulmonary infections in immunocompromised patients. However, MRI can be used in the follow-up imaging of these patients.

Key words: lung MRI, immunocompromised host, pulmonary infections.

\section{Introduction}

There has been a continuous rise in the number of immunocompromised patients secondary to the advancement in the treatment of cancer, autoimmune disorders, and an increase in the number of organ/stem cell transplant recipients. In developing nations like India, acquired immunodeficiency syndrome (AIDS) has also led to an increasing number of immunocompromised patients $[1,2]$. Pulmonary infections account for approximately $75 \%$ of all pulmonary complications in these patients [3]. Sero- logical and microbiological culture tests are of limited use in many of these cases [4,5]. A timely diagnosis of these uncommon infections is important for the early initiation of adequate treatment $[6,7]$.

Chest radiograph (CXR) is usually the first radiological investigation performed in these patients. Its sensitivity has been shown to range from $20 \%$ to $38 \%$, as shown in different studies [8-10]. High-resolution computed tomography (HRCT) has greater sensitivity and specificity in detecting pulmonary pathologies, as compared to CXR [11-13]. However, immunocompromised patients are

\section{Correspondence address:}

Prof. Mandeep Garg, Post Graduate Institute of Medical Education and Research, 160012, Chandigarh, India, e-mail: gargmandeep@hotmail.com Authors' contribution:

A Study design · B Data collection · C Statistical analysis · D Data interpretation · E Manuscript preparation · F Literature search · G Funds collection 
prone to develop recurrent episodes of infections, thereby requiring repeated CT examinations, which lead to an undesirably high cumulative radiation exposure.

Magnetic resonance imaging (MRI), being radiation free, is emerging as a new diagnostic modality in thoracic imaging. The lung is predominantly composed of air and has a low density of hydrogen protons. Hence, the MR signal generated from the lung parenchyma is poor. Infections result in an increase in the water content within the involved lung parenchyma resulting in bright signal on MRI due to an increase in the proton density. Recent developments in MRI techniques have allowed imaging of lung tissue with better image quality [14-16]. There are studies on lung MRI that have used HASTE and volumetric interpolated breath-hold examination (VIBE) MR sequences [15,17-22]. Other studies have used other MRI sequences like SPAIR, short tau inversion recovery (STIR), and T1-weighted fast field echo (T1-FFE) [23]. There is a need for lung MR sequences that have short acquisition time, better image quality, and minimal artefacts. In this study, we have evaluated the diagnostic accuracy of MRI, using different pulse sequences, in pulmonary infections in immunocompromised adults. Reconstructed HRCT images have been considered as the gold standard.

\section{Material and methods}

This prospective study on 35 immunocompromised patients was conducted from January 2016 to May 2017. Approval was obtained from the institute Ethics Committee, and informed written consent was taken from all patients. Inclusion criteria were immunocompromised adults (e.g. patients receiving immunosuppressive therapy, cancer chemotherapy, neutropaenic patients) with persisting fever and clinical suspicion of pulmonary infection, who presented to our department for a CT scan. Exclusion criteria were contraindications to MRI (claustrophobia, potentially magnetic implants, etc.), the time interval of more than 48 hours between MRI and CT scan, and age less than 18 years.

\section{Computed tomography protocol}

Non-contrast CT was performed with the patient in a supine position, and sections were taken from lung apices to the domes of diaphragm using a dual-source CT 128 slice scanner - Somatom Definition Flash (Siemens Healthcare, Forchheim, Germany)/third-generation 16 slice scanner, CT sensation 16 (Siemens Healthcare, Forchheim, Germany)/CT 256 Slice scanner, Philips Brilliance iCT (Philips Healthcare Best, The Netherlands). A helical dataset of the chest was acquired with $10 \mathrm{~mm}$ thick sections at $10 \mathrm{~mm}$ increment with automatic exposure control. HRCT images were reconstructed from the helical dataset into $1 \mathrm{~mm}$ sections at $1 \mathrm{~mm}$ increment (reconstruction kernel: B $80 \mathrm{f}$ ultrasharp filter for lung window and B 30 f medium smooth for mediastinal window) using filter back projection.

\section{Magnetic resonance imaging protocol}

All the patients were examined using a 1.5 Tesla MRI unit (MAGNETOM AERA; Siemens Medical Solutions, Malvern, $\mathrm{Pa}$ ). These patients underwent MR scanning of the thorax from lung apices to the domes of the diaphragm.

The following sequences were utilised: axial HASTE (breath-hold), coronal HASTE (breath-hold), TRUFI (breath-hold), SPAIR (breath-hold), 3D FLASH (breathhold), and BLADE (respiratory triggered). No gadolinium-based contrast agents were administered. MRI examination was completed within five minutes. Imaging parameters are given in Table 1.

Table 1. Imaging parameters of various magnetic resonance images sequences used during study

\begin{tabular}{|c|c|c|c|c|c|c|}
\hline & \multicolumn{2}{|c|}{ T2 HASTE ${ }^{\alpha}$} & \multirow[t]{2}{*}{ SPAIR $^{\beta}$} & \multirow[t]{2}{*}{ 3D FLASH $x$} & \multirow[t]{2}{*}{$\mathrm{TRUFI}^{\delta}$} & \multirow[t]{2}{*}{ BLADE $^{\varepsilon}$} \\
\hline & Axial & Coronal & & & & \\
\hline $\mathrm{TR} / \mathrm{TE}$ (ms) & $500 / 39$ & $1000 / 9$ & $500 / 39$ & $2.6 / 0.94$ & $448 / 144$ & $2500 / 63$ \\
\hline Slice thickness (mm) & 5 & 6 & 5 & 2.2 & 5 & 6 \\
\hline Slice per station $(n)$ & 30 & 25 & 30 & 112 & 30 & 30 \\
\hline FOV (mm) & 320 & 350 & 320 & 350 & 320 & 320 \\
\hline Acquired voxel size $\left(\mathrm{mm}^{3}\right)$ & $1.3 \times 1.3 \times 5$ & $1.1 \times 1.1 \times 6$ & $1.3 \times 1.3 \times 5$ & $1.2 \times 1.2 \times 2.2$ & $0.6 \times 0.6 \times 0.6$ & $1.3 \times 1.3 \times 6$ \\
\hline Bandwidth/pixel (Hz) & 781 & 781 & 781 & 640 & 1028 & 501 \\
\hline Signal averages $(n)$ & 2 & 1 & 2 & 1 & 2 & 1 \\
\hline Flip angle (degree) & 146 & 150 & 148 & 20 & 70 & 150 \\
\hline Acquisition time & $30 \mathrm{sec}$ & $30 \mathrm{sec}$ & $30 \mathrm{sec}$ & $16 \mathrm{sec}$ & $27 \mathrm{sec}$ & $72 \mathrm{sec}$ \\
\hline
\end{tabular}

${ }^{*}$ Repetition time/Echo time, FOV - field of view. ${ }^{\alpha}$ Half-Fourier acquisition single-shot turbo spin-echo. ${ }^{\beta}$ Spectrally attenuated inversion recovery. ${ }^{\times 3}$-Dimensional fast low-angle shot. ${ }^{\top}$ True fast

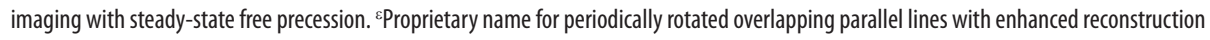




\section{Image evaluation}

All CT scans were evaluated at various workstations after reconstruction (Wizard workstation for CT sensation 16; at Syngo.via workstation for Somatom Definition Flash; at Extended Brilliance Workspace for 256 slice Phillips Brilliance ICT). Monitors with window settings suitable for lung parenchyma (window width $1200 \mathrm{HU}$; window level $-600 \mathrm{HU}$ ) and for soft tissue/mediastinum (window width $400 \mathrm{HU}$; window level $40 \mathrm{HU}$ ) were used.

Two experienced chest radiologists evaluated the CT and MRI scans independently. The radiologists were blinded to each other's observations. CT findings were taken as the reference standard. The lungs were evaluated for nodules, consolidation, and ground-glass opacities (GGOs). Nodules were categorised as solitary, few (2-5), and multiple $(>5)$ and were evaluated for their size, the presence of a halo sign, cavitation, and calcification. Nodule size was measured by taking the largest diameter as seen in the lung window. Nodules were divided into two categories based on their size: $<5 \mathrm{~mm}$ and $\geq 5 \mathrm{~mm}$. All MRI sequences and their images were also scrutinised for the above-mentioned findings.

The MR images of each sequence were evaluated for quality, and scoring was performed as follows: 1 - no artefacts; 2 - minimal artefacts; 3 - interpretable but impaired by artefacts; 4 - not interpretable. Ancillary findings such as pleural effusion, lymph nodes, etc. were also reported separately.

\section{Statistical analysis}

The sensitivity, specificity, positive predictive value (PPV), and negative predictive value (NPV) of the various MRI sequences were calculated. Concordance and discordance were calculated by the Kappa test. McNemar's test was used to see differences between the two modalities. All the statistical tests were performed at a significance level of $\alpha=0.05$ and were two-sided. All statistical analysis was performed using SPSS (IBM SPSS Statistics for Windows, Version 22.0.0, IBM Corporation, Armonk, NY, USA).

\section{Results}

The mean age of the included patients was 40.3 years, with 20 males and 15 females. Our study included 15 post-renal transplant patients, five cases of carcinoma ovary on chemotherapy, two patients were human immunodeficiency virus (HIV) positive, three had a history of chronic steroid intake, eight patients were suffering from lymphoma, one had acute myeloid leukaemia (AML), and one was a case of bone marrow transplant on chemotherapy. All CT and MRI scans were done without any complications. A patient-to-patient comparison of the findings of $\mathrm{CT}$ and MRI was done. Perfect agreement was observed between the two radiologists.
Table 2. Table depicting frequency distribution of score for different magnetic resonance images sequences

\begin{tabular}{lc|c|c|c|} 
& \multicolumn{2}{c|}{ Score 2* $^{*}$} & \multicolumn{2}{c}{ Score 3* $^{*}$} \\
\cline { 2 - 5 } & Count & $\%$ & Count & $\%$ \\
\hline HASTE $^{\alpha}$ coronal & 2 & 5.7 & 33 & 94.3 \\
\hline HASTE $^{\alpha}$ axial & 35 & 100.0 & - & - \\
TRUFF $^{\delta}$ & 33 & 94.3 & 2 & 5.7 \\
SPAIR $^{\beta}$ & 31 & 88.6 & 4 & 11.4 \\
3D FLASH $^{\alpha}$ & 1 & 2.9 & 34 & 97.1 \\
BLADE $^{\varepsilon}$ & 22 & 62.9 & 13 & 37.1 \\
\hline
\end{tabular}

${ }^{\alpha}$ Half-Fourier acquisition single-shot turbo spin-echo. ${ }^{\beta}$ Spectrally attenuated inversion recovery. ${ }^{\delta}$ True fast imaging with steady-state free precession. ${ }^{\times 3}$-Dimensional fast low-angle shot. ${ }^{.}$Proprietary name for periodically rotated overlapping parallel lines with enhanced reconstruction *None of the MR sequences had quality score of 1 or 4

\section{Computed tomography findings}

Nine (25.7\%) patients showed areas of consolidations, nine $(25.7 \%)$ patients showed GGOs, and 14 (40\%) patients showed nodules. CT was normal in three patients. In eight patients, other findings like pleural effusion, mediastinal lymphadenopathy, etc. were seen and recorded as ancillary findings.

\section{Magnetic resonance imaging findings}

There were no MR images with sufficient artefacts to make them diagnostically unacceptable (score 4) as well as no MR images without any artefacts (score 1). Frequency distribution of score 2 and score 3 for different MRI sequences have been elaborated in Table (Table 2). Ten (28.5\%) patients showed areas of consolidations, 13 (37.1\%) patients showed GGOs, and nine (25.7\%) patients showed nodules. MRI was normal in seven patients. In 11 patients, other findings like pleural effusion, mediastinal lymphadenopathy, etc. were seen and recorded as ancillary findings.

\section{Correlation between computed tomography and magnetic resonance imaging findings}

MRI was negative in five patients in whom CT showed findings. Nodules were present in 14 patients on CT and nine patients on MRI. Nodules of size less than $5 \mathrm{~mm}$ were present in nine patients on CT and one patient on MRI. Nodules of size more than $5 \mathrm{~mm}$ were present in eight patients on CT and eight patients on MRI. Halo sign and cavitating nodule were positive in two patients on CT and three patients on MRI. Consolidation was present in nine patients on CT and 10 patients on MRI. Consolidation with breakdown was seen in two patients on CT and in three patients on MRI. GGOs were seen in nine patients on CT and in 13 patients on MRI (Table 3). Kappa test revealed moderate agreement between CT and MRI scans for the nodules and GGO detection $(\kappa=0.48$ for 
Table 3. Table depicting diagnostic accuracy of magnetic resonance images in relation to various lung findings

\begin{tabular}{|l|c|c|c|c|c|c|c|c|}
\hline Parameter & TP & TN & FP & FN & Sensitivity & Specificity & NPV & PPV \\
\hline Nodule, overall & 7 & 19 & 2 & 7 & $50 \%$ & $90.4 \%$ & $73 \%$ & $77.7 \%$ \\
\hline$<5 \mathrm{~mm}$ & 1 & 26 & 0 & 8 & $11.1 \%$ & $100 \%$ & $76.4 \%$ & $100 \%$ \\
\hline$>5 \mathrm{~mm}$ & 6 & 25 & 2 & 2 & $75 \%$ & $92.5 \%$ & $92.5 \%$ & $75 \%$ \\
\hline Halo sign & 2 & 32 & 1 & 0 & $100 \%$ & $96.9 \%$ & $100 \%$ & $66.6 \%$ \\
\hline Cavitating nodule & 2 & 32 & 1 & 0 & $100 \%$ & $96.9 \%$ & $100 \%$ & $66.6 \%$ \\
\hline consolidation & 9 & 25 & 1 & 0 & $100 \%$ & $96 \%$ & $100 \%$ & $90 \%$ \\
\hline Consolidation with breakdown & 2 & 32 & 1 & 0 & $100 \%$ & $96.9 \%$ & $100 \%$ & $66.6 \%$ \\
\hline GGOs & 7 & 20 & 6 & 2 & $77.7 \%$ & $76.9 \%$ & $90.9 \%$ & $53.8 \%$ \\
\hline
\end{tabular}

TP - true positives, TN - true negatives, FP - false positives, FN - false negatives, NPV - negative predictive value, PPV - positive predictive value, GGOs - ground-glass opacities

nodule and $\kappa=0.55$ for GGOs). Excellent agreement was seen between CT and MRI scan for consolidation detection, with a $\kappa$ value of 0.839 . Kappa test revealed good agreement between CT and MRI scan for the cavitating nodule, halo sign, and consolidation with breakdown
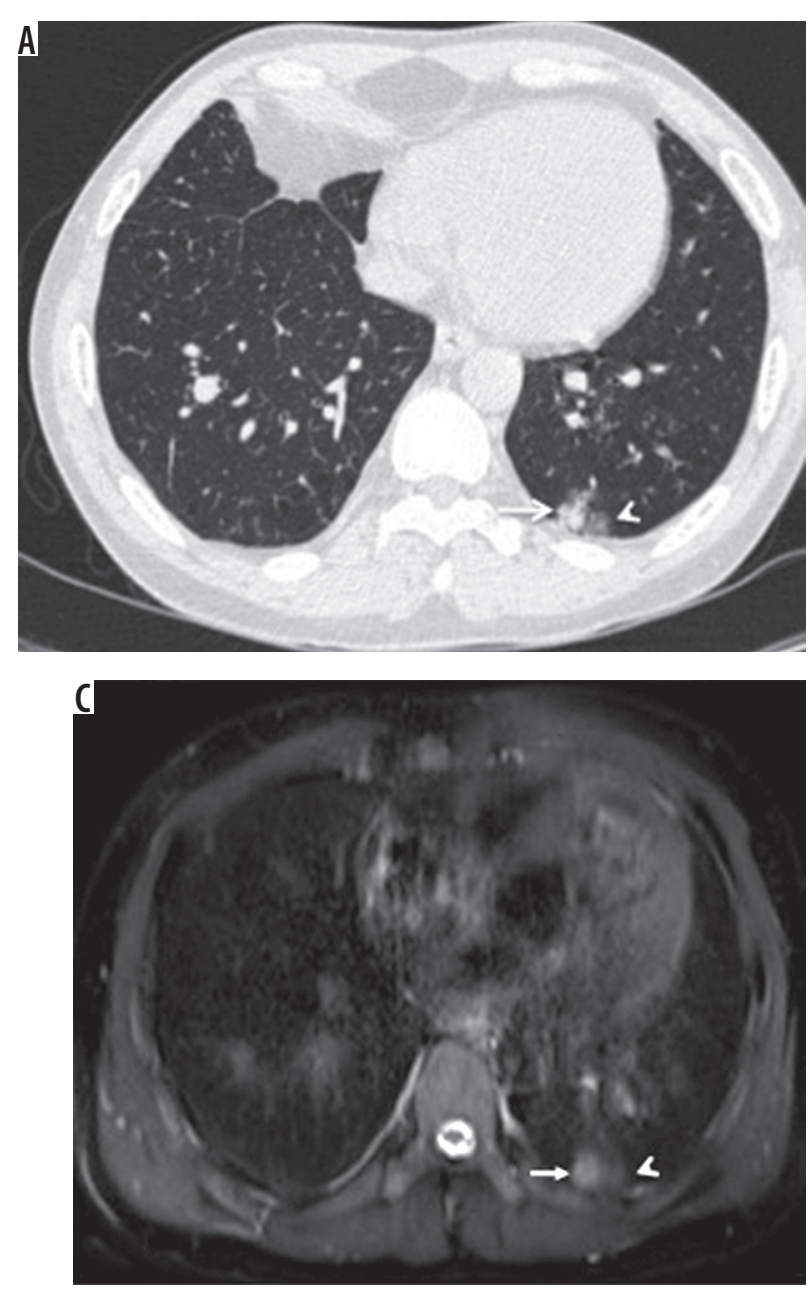

detection, with $\kappa$ values of $0.785,0.785$, and 0.785 , respectively. Overall, no significant difference was observed between CT and MRI by the McNemar test $(p>0.05)$ for the detection of consolidation. Strong agreement was found between the two observers for CT and MRI findings based

Figure 1. A) HRCT, B) TRUFI, C) SPAIR, and D) HASTE - axial images of a 30-year-old male patient with lymphoma, on chemotherapy, complaining of cough and fever. Computed tomography image shows a well-defined nodule (arrow) with surrounding ground-glass opacities (arrowhead) in the posterobasal segment of the left lower lobe, which is also well demonstrated on magnetic resonance images 

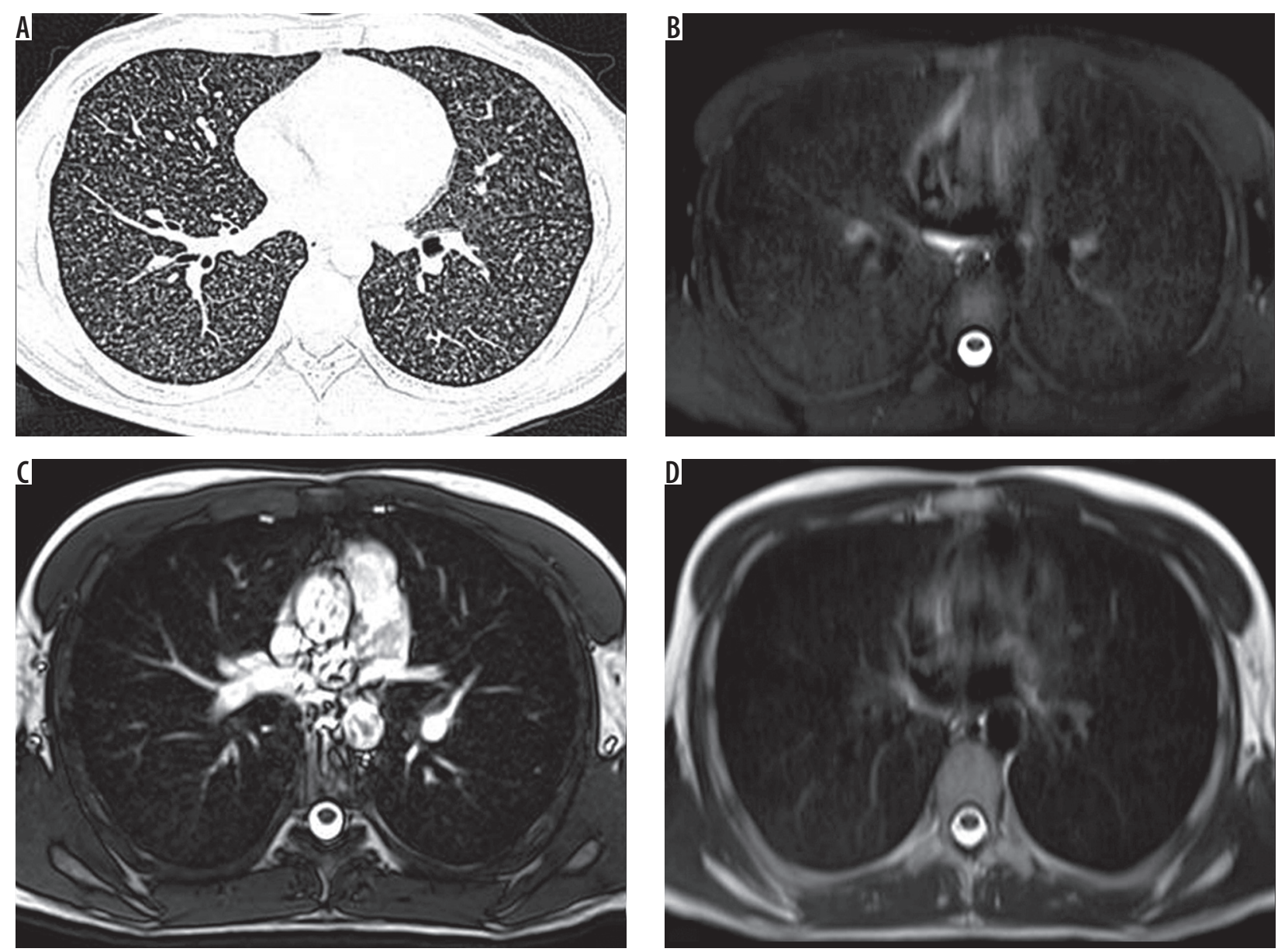

Figure 2. A) HR(T, B) SPAIR, C) TRUFI, and D) BLADE - axial images of a 26-year-old male patient known case of lymphoma, on chemotherapy, complaining of cough and fever. Computed tomography shows miliary nodules in bilateral lungs. SPAIR magnetic resonance images show diffusely increased signal intensity with no discrete nodules. On TRUFI and BLADE, no abnormality is demonstrated

on the $\kappa$ test result (minimum $\kappa=0.86$ and maximum $\kappa=1$ ) (Figures 1-4).

\section{Analysis of different magnetic resonance pulse sequences}

Nodules were detected best on the T2 HASTE axial, SPAIR MR pulse sequences, and least detected on the T2 HASTE COR sequence. Halo sign was seen in T2 HASTE axial, SPAIR, and BLADE sequence. It could not be detected on T2 HASTE COR, TRUFI, and 3D FLASH sequences. TRUFI and BLADE sequences detected cavitating nodule and consolidation with $100 \%$ sensitivity. Consolidation with breakdown was seen in all sequences. SPAIR sequence had the highest rate of GGO detection but with the highest false positivity. T2 HASTE axial sequence had sensitivity similar to the SPAIR sequence with a less false-positive rate. GGOs could not be detected on the T2 HASTE COR sequence (Table 4).

\section{Discussion}

The number of immunocompromised patients has risen due to evolving expertise in the clinical management of malignancies and organ transplantation. These patients are at a higher risk of acquiring repeated lung infections and usually present with fever. CT chest is the investigation of choice in these patients for the early diagnosis of lung infection and for the treatment response evaluation. However, it leads to significant radiation exposure to the patient. Despite various measures to minimise radiation exposure in these patients and use of low-dose CT protocols, there remains a radiation exposure hazard due to repeated examinations. Radiation exposure of $10 \mathrm{mSV}$ carries a 1/2000 possibility of the development of fatal cancer during the lifetime of a patient [24]. Hence, there is a strong need for an alternate radiation-free imaging modality that could provide the same information as a CT scan to evaluate these patients for pulmonary infections.

In the present study, we modified MRI sequences to detect the findings with the minimum possible scanning duration. We brought down the total scan duration to 3 minutes 25 seconds, and hence we could achieve 100\% feasibility of performing lung MRI.

The overall sensitivity of MRI for detection of lung nodule was $50 \%$ in our study. This low sensitivity was 

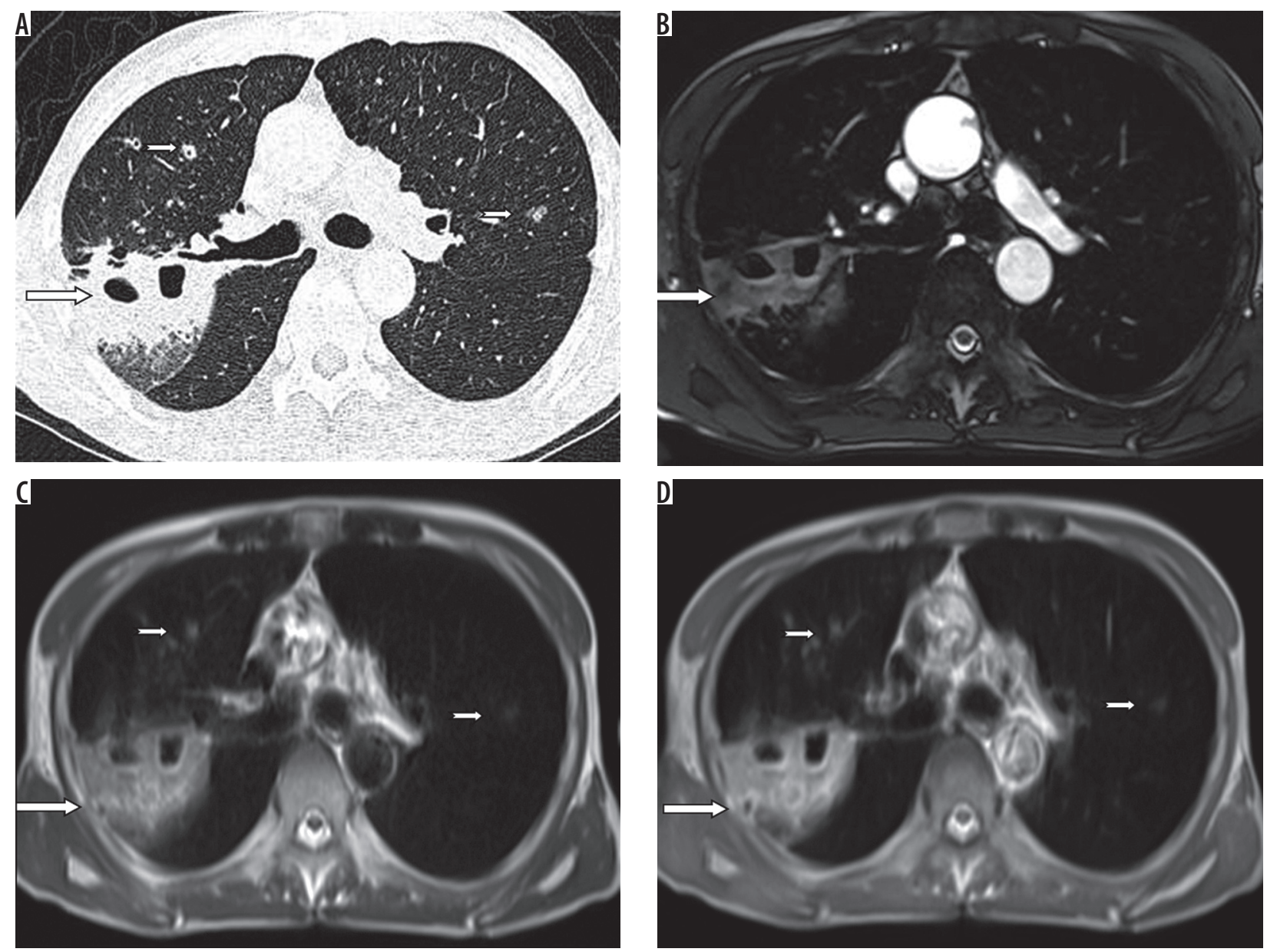

Figure 3. A) HRCT, B) TRUFI, C) BLADE, and D) HASTE - axial images of a 48-year-old female patient of carcinoma ovary, on chemotherapy, complaining of cough and fever. Computed tomography shows a patchy area of consolidation (long arrows) with breakdown in the posterior segment of the right upper lobe and few nodules (short arrows) in the anterior segment of right upper lobe and the apicoposterior segment of left upper lobe, which are also well demonstrated on BLADE and HASTE images. On TRUFI sequence, nodules in the left upper lobe and right upper lobe are not demonstrated

probably due to the fact that the majority of the detected nodules were of small size. This is evident from the fact that the sensitivity for the detection of nodules increased from $11.1 \%$ to $75 \%$ as the size of the nodules increased. This difference can also be attributed to the different thickness of the section used for CT $(0.1 \mathrm{~cm})$ and MR imaging $(0.5-0.6 \mathrm{~cm})$. This result is in concordance to the previous studies published by Eibel et al. and Ekinci et al. $[15,22]$. Most of the previous studies acquired CT images at a $10 \mathrm{~mm}$ increment. In our study, a helical dataset of the NCCT chest was acquired, and it was further reconstructed into $1 \mathrm{~mm}$ sections at a $1 \mathrm{~mm}$ increment, thereby detecting a larger number of smaller nodules than in previous studies. This could also be one of the reasons for less MR sensitivity as compared to previous studies. In this study, the presence of centrilobular nodules and nodules less than $5 \mathrm{~mm}$ were missed on MRI. In one patient, miliary nodules were also missed on MRI.

MRI was found to be $100 \%$ sensitive with PPV of $66.6 \%$ for the detection of halo sign in our study, which is in agreement with the previous study done by Claudia et al. [19]. In one patient halo sign was reported on MRI but not on CT scan, which may be due to blurring artefact on MRI which was misinterpreted as halo sign.

For the detection of the cavitating nodules, MRI was found to be $100 \%$ sensitive with PPV of $66.6 \%$ in our study. This result is similar to the previous studies done by Claudia et al. and Sodhi et al. but is in contrast to the previous studies done by Eibel et al. [15,19-21]. In one patient, a cavitating nodule was seen on MRI but was not present on CT. In this patient, the interval between CT and MR was 36 hours. Probably, due to the longer time interval, cavitation developed in the nodule, which was picked up on MR but was not present at the time of CT acquisition.

The result for the detection of consolidation and consolidation with a breakdown on MRI was similar to the other previous studies with a sensitivity of $100 \%$ [20-22].

For the detection of GGOs, CT is far better than MRI. In our study, GGOs were detected on MRI with sensitivity, specificity, PPV, and NPV of 77.7\%, 76.9\%, 53.8\%, and $90.9 \%$, respectively, with MRI showing a high false-positive rate. This result is also similar to previous studies [20-22]. GGOs are mainly due to either thickening of 

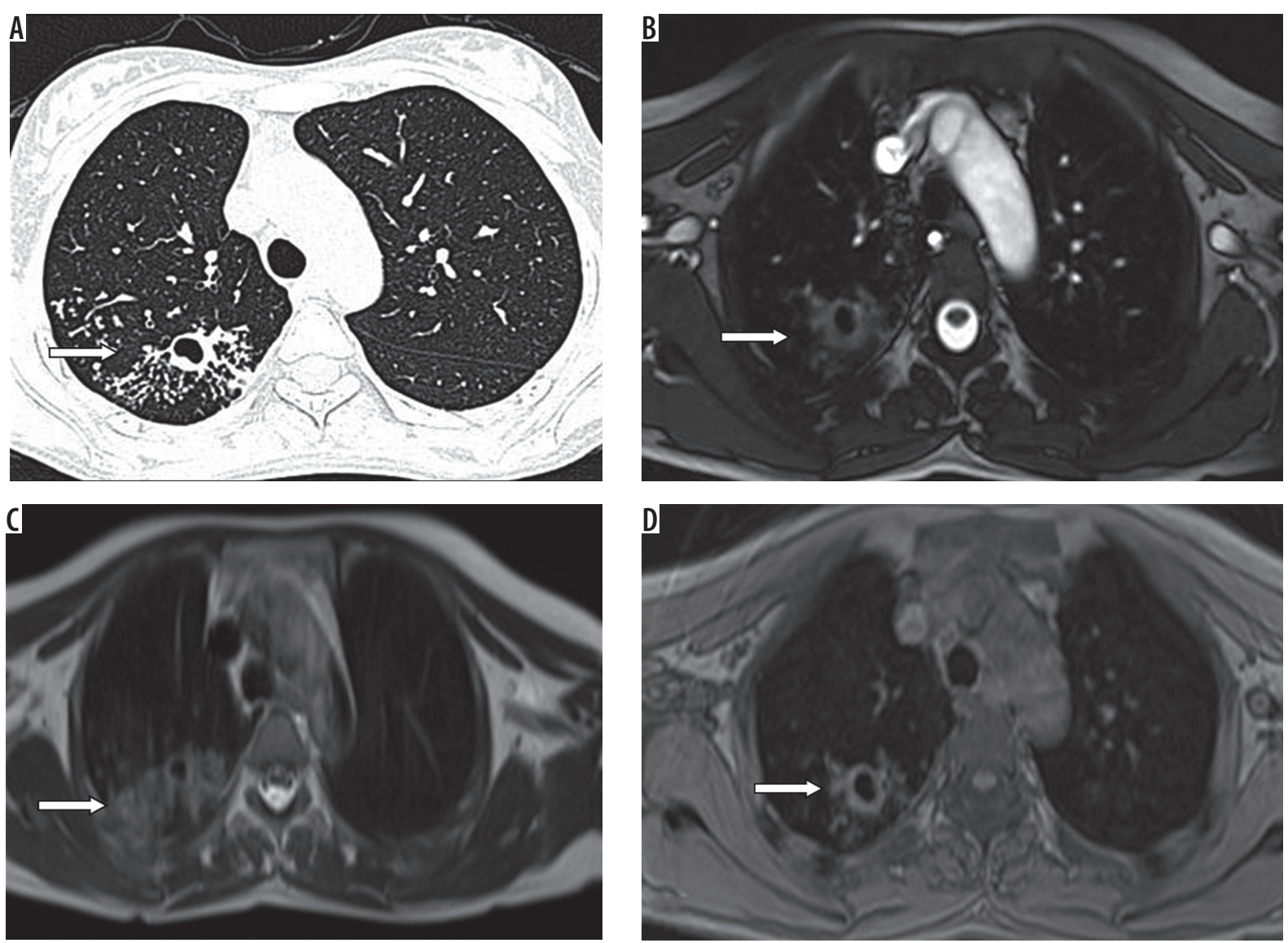

Figure 4. A) HR(T, B) TRUFI, C) BLADE, and D) VIBE - axial images of an 18-year-old female patient with a history of renal transplantation, on immunosuppressive drugs, complaining of cough and fever. HRCT chest image shows a cavitating nodule with adjacent centrilobular nodules (arrows) in the posterior segment of the right upper lobe, which is also well demonstrated on magnetic resonance images

interlobular/intralobular interstitium of the acini or due to cells and fluid filling the lung acini. These findings are usually subtle, and low sensitivity may be secondary to the low signal strength generated from the proton-sparse lung parenchyma. The currently available MR technology does not have the resolution to detect such signal alterations. This may also be a cause for the high false-negative rate for GGO detection on MRI. The explanation for the high false-positive rate could be the blurring and ghost artefact on MRI, which resembles GGOs.

For the detection of the nodules, halo sign, cavitating nodule, and consolidation with breakdown T2 HASTE axial, and SPAIR and BLADE sequences showed similar sensitivity. But, scanning time for T2 HASTE axial and SPAIR was only 30 seconds each, while the scanning time for the BLADE sequence was 72 seconds. So, the BLADE sequence did not add any additional information to the study despite increasing the duration of the scan. Consolidation was seen on almost all sequences with almost similar detection rates. There was one false-positive consolidation patch on T2 HASTE axial and SPAIR sequences. This was due to the clustering of multiple nodules, which erroneously appeared as a patch of consolidation. And as discussed previously, for detection of GGOs, MRI showed poor sensitivity, specificity, PPV, and NPV. Of the five sequences, the SPAIR sequence has the highest false-positive rate, but it was found to be the most sensitive sequence for the detection of GGOs. BLADE had the lowest false-positive rate. T2 HASTE coronal and 3D FLASH sequence showed the maximum artefacts and did not pick up most of the findings. Thus, these sequences are of limited diagnostic importance in lung MRI imaging.

There are few limitations in our study. First it is done on a relatively small sample size. Second, a non-contrast MRI study was done, so we could not analyse the role of contrast enhancement on nodule detection in this study. Third, we did not find any nodules with calcification in our study. Hence, this character of the nodule could not be evaluated in our study.

\section{Conclusions}

Lung MRI has sensitivity similar to chest CT for the detection of consolidation. Lung MRI has poor sensitivity for the detection of nodules, and this sensitivity is further decreased for nodules of size less than $5 \mathrm{~mm}$. Lung MRI also has a high false-positive rate for the detection of GGOs. As small nodules and GGOs are the most common 
Table 4. Table depicting diagnostic accuracy of various magnetic resonance image pulse sequences

\begin{tabular}{|c|c|c|c|c|c|c|}
\hline Parameter & $\begin{array}{c}\text { HASTE }^{\alpha} \\
\text { axial }\end{array}$ & $\begin{array}{l}\text { HASTE }^{\alpha} \\
\text { coronal }\end{array}$ & TRUF $^{\delta}$ & SPAIR $^{\beta}$ & 3D FLASH $x$ & BLADE $^{\varepsilon}$ \\
\hline \multicolumn{7}{|l|}{ HALO sign } \\
\hline Sensitivity & $100 \%$ & * & * & $100 \%$ & * & $100 \%$ \\
\hline Specificity & $96.9 \%$ & $100 \%$ & $100 \%$ & $96.9 \%$ & $100 \%$ & $96.9 \%$ \\
\hline NPV & $100 \%$ & $94.2 \%$ & $94.2 \%$ & $100 \%$ & $94.2 \%$ & $100 \%$ \\
\hline PPV & $66.6 \%$ & * & * & $66.6 \%$ & * & $66.6 \%$ \\
\hline \multicolumn{7}{|l|}{ Nodules } \\
\hline Sensitivity & $50 \%$ & $7 \%$ & $42.8 \%$ & $50 \%$ & $28.5 \%$ & $50 \%$ \\
\hline Specificity & $95.2 \%$ & $100 \%$ & $95.2 \%$ & $95.2 \%$ & $100 \%$ & $90.4 \%$ \\
\hline NPV & $74 \%$ & $61.7 \%$ & $85.7 \%$ & $74 \%$ & $67.7 \%$ & $73 \%$ \\
\hline PPV & $87.5 \%$ & $100 \%$ & $71.4 \%$ & $87.5 \%$ & $100 \%$ & $77.7 \%$ \\
\hline \multicolumn{7}{|c|}{ Cavitating nodule } \\
\hline Sensitivity & $100 \%$ & * & $100 \%$ & $100 \%$ & $50 \%$ & $100 \%$ \\
\hline Specificity & $96.9 \%$ & $100 \%$ & $100 \%$ & $96.9 \%$ & $100 \%$ & $100 \%$ \\
\hline NPV & $100 \%$ & $94.2 \%$ & $100 \%$ & $100 \%$ & $97 \%$ & $100 \%$ \\
\hline PPV & $66.6 \%$ & * & $100 \%$ & $66.6 \%$ & $100 \%$ & $100 \%$ \\
\hline \multicolumn{7}{|l|}{ Consolidation } \\
\hline Sensitivity & $100 \%$ & $77 \%$ & $100 \%$ & $100 \%$ & $77 \%$ & $100 \%$ \\
\hline Specificity & $96 \%$ & $100 \%$ & $100 \%$ & $96 \%$ & $100 \%$ & $100 \%$ \\
\hline NPV & $100 \%$ & $92.8 \%$ & $100 \%$ & $100 \%$ & $92.8 \%$ & $100 \%$ \\
\hline PPV & $90 \%$ & $100 \%$ & $100 \%$ & $90 \%$ & $100 \%$ & $100 \%$ \\
\hline \multicolumn{7}{|c|}{ Consolidation with breakdown } \\
\hline Sensitivity & $100 \%$ & $50 \%$ & $100 \%$ & $100 \%$ & $50 \%$ & $100 \%$ \\
\hline Specificity & $96.9 \%$ & $96.9 \%$ & $96.9 \%$ & $96.9 \%$ & $96.9 \%$ & $96.9 \%$ \\
\hline NPV & $100 \%$ & $96.9 \%$ & $100 \%$ & $100 \%$ & $96.9 \%$ & $100 \%$ \\
\hline PPV & $66.6 \%$ & $50 \%$ & $66.6 \%$ & $66.6 \%$ & $50 \%$ & $66.6 \%$ \\
\hline \multicolumn{7}{|l|}{$\mathrm{GGOS}$} \\
\hline Sensitivity & $66.6 \%$ & * & $16.6 \%$ & $77.7 \%$ & $33.3 \%$ & $55.5 \%$ \\
\hline Specificity & $88.4 \%$ & $92.8 \%$ & $86.2 \%$ & $76.9 \%$ & $81.2 \%$ & $96.1 \%$ \\
\hline NPV & $88.4 \%$ & $78.7 \%$ & $83.3 \%$ & $90.9 \%$ & $92.8 \%$ & $86.2 \%$ \\
\hline PPV & $66.6 \%$ & * & $20 \%$ & $53.8 \%$ & $14.2 \%$ & $83.3 \%$ \\
\hline
\end{tabular}

${ }^{\alpha}$ Half-Fourier acquisition single-shot turbo spin-echo. ${ }^{\delta}$ True fast imaging with steady-state free precession. ${ }^{B S p e c t r a l l y ~ a t t e n u a t e d ~ i n v e r s i o n ~ r e c o v e r y . ~}{ }^{\times 3-D i m e n s i o n a l ~ f a s t ~ l o w-a n g l e ~ s h o t . ~}{ }^{\text {EPropri- }}$ etary name for periodically rotated overlapping parallel lines with enhanced reconstruction. *Not detected in this sequence. NPV - negative predictive value, PPV - positive predictive value, GGOS - ground-glass opacities

findings in immunocompromised adults CT scan remains the imaging modality for the evaluation of pulmonary infections in these patients. Respiratory triggered MR pulse sequences did not add any significant additional diagnostic information as compared to the non-respiratory-triggered MRI pulse sequences for the detection of nodules, consolidation, and ground-glass opacities. T2 HASTE axial and BLADE sequences are the most important sequences for pulmonary parenchymal pathology detection.

\section{Conflict of interest}

The authors report no conflict of interest. 


\section{References}

1. Rubin RH, Peterson PK. Overview of pneumonia in the compromised host. Semin Respir Infect 1986; 1: 131-132.

2. Crothers K, Thompson BW, Burkhardt K, et al. HIV-associated lung infections and complications in the era of combination antiretroviral therapy. Proc Am Thorac Soc 2011; 8: 275-281.

3. Rosenow EC, Wilson WR, Cockerill FR. Pulmonary disease in the immunocompromised host. 1. Mayo Clin Proc 1985; 60: 473-487.

4. Walsh TJ, Anaissie EJ, Denning DW, et al. Treatment of aspergillosis: clinical practice guidelines of the Infectious Diseases Society of America. Clin Infect Dis 2008; 46: 327-360.

5. Maschmeyer G, Haas A, Cornely OA. Invasive aspergillosis: epidemiology, diagnosis, and management in immunocompromised patients. Drugs 2007; 67: 1567-1601.

6. Ewig S, Glasmacher A, Ulrich B, et al. Pulmonary infiltrates in neutropenic patients with acute leukemia during chemotherapy: outcome and prognostic factors. Chest 1998; 114: 444-451.

7. Maschmeyer G, Link H, Hiddemann W, et al. Pulmonary infiltrations in febrile patients with neutropenia. Risk factors and outcome under empirical antimicrobial therapy in a randomized multicenter study. Cancer 1994; 73: 2296-2304.

8. Donowitz GR, Harmann C, Pope T, et al. The role of chest roentgenogram in febrile neutropenic patients. Arch Intern Med 1991; 151: 701-704.

9. Roy V, Ali LI, Selby GB. Routine chest radiography for the evaluation of febrile neutropenic patients after autologous stem cell transplantation. Am J Hematol 2000; 64: 170-174.

10. Katz JA, Bash R, Rollins N, et al. The yield of routine chest radiography in children with cancer hospitalized for fever and neutropenia. Cancer 1991; 68: 940-943.

11. Haugaard A, Ellis M, Ekelund L. Early chest radiography and CT in the diagnosis, management, and outcome of invasive pulmonary aspergillosis. Acta Radiol 2002; 43: 292-298.

12. Heussel CP, Kauczor HU, Heussel GE, et al. Pneumonia in febrile neutropenic patients in bone marrow and blood stem-cell transplant recipients: use of high-resolution computed tomography. J Clin Oncol 1999; 17: 796-805.

13. Kang M, Deoghuria D, Varma S, et al. Role of HRCT in detection and characterization of pulmonary abnormalities in patients with febrile neutropenia. Lung India 2013; 1: 124-130.

14. Biederer J, Busse I, Grimm J, et al. Sensitivity of MRI in detecting alveolar Infiltrates: experimental studies. ROFO 2002; 174: 10331039.
15. Eibel R, Herzog P, Dietrich O, et al. Pulmonary abnormalities in immunocompromised patients: comparative detection with parallel acquisition MR imaging and thin-section helical CT. Radiology 2006; 241: 880-891.

16. Moody AR, Bolton SC, Horsfield MA. Optimization of a breathhold magnetic resonance gradient echo technique for the detection of interstitial lung disease. Invest Radiol 1995; 30: 730-737.

17. Rieger C, Herzog P, Eibel R, et al. Pulmonary MRI - a new approach for the evaluation of febrile neutropenic patients with malignancies. Support Care Cancer 2008; 16: 599-606.

18. Attenberger UI, Morelli JN, Henzler T, et al. 3Tesla proton MRI for the diagnosis of pneumonia/lung infiltrates in neutropenic patients with acute myeloid leukemia: initial results in comparison to HRCT. Eur J Radiol 2014; 83: e61-66.

19. Leutner CC, Gieseke J, Lutterbey G, et al. MR imaging of pneumonia in immunocompromised patients: comparison with helical CT. AJR Am J Roentgenol 2000; 175: 391-397.

20. Sodhi KS, Khandelwal N, Saxena AK, et al. Rapid lung MRI - paradigm shift in evaluation of febrile neutropenia in children with leukemia: a pilot study. Leuk Lymphoma 2016; 57: 70-75.

21. Sodhi KS, Khandelwal N, Saxena AK, et al. Rapid lung MRI in children with pulmonary infections: time to change our diagnostic algorithms: rapid lung MRI in children. J Magn Reson Imaging 2016; 43: 1196-1206.

22. Ekinci A, Yucel Ucarkus T, Okur A, et al. MRI of pneumonia in immunocompromised patients: comparison with CT. Diagn Interv Radiol 2017; 23: 22-28.

23. Yan C, Tan X, Wei Q, et al. Lung MRI of invasive fungal infection at 3 Tesla: evaluation of five different pulse sequences and comparison with multidetector computed tomography (MDCT). Eur Radiol 2015; 25: 550-557.

24. Committee to Assess Health Risks from Exposure to Low Levels of Ionizing Radiation; Nuclear and Radiation Studies Board, Division on Earth and Life Studies, National Research Council of the National Academies. Health risks from exposure to low levels of ionizing radiation: BEIR VII Phase 2. Washington, DC: The National Academies Press; 2006.17 